\title{
Exergy Applied to Lunar Base Design
}

\author{
Mark B. Luther ${ }^{1}$ \\ Deakin University, Geelong, Victoria, 3217, Australia \\ and \\ Theodore W. Hall ${ }^{2}$ \\ University of Michigan, Ann Arbor, Michigan, 48109, USA
}

\begin{abstract}
This paper explores design considerations for energy efficiency in lunar habitats. It considers several previous lunar energy studies in regards to energy types and stages of energy requirements. If we are to obtain true sustainability in energy processes, we will need to design according to the principles "exergy", considering both the first and the second laws of thermodynamics in a holistic and thorough evaluation of energy capture, transformation, and use. Such an evaluation will ascertain the source of energy, its processing and energy potential stages, as well as the task required. Traditional designs of facility thermal systems are frequently extremely wasteful: they dramatically increase both first costs and operating costs because they treat heating and cooling systems as separate entities, instead of an integrated energy system. Energy processes, the state of energy required to do a particular task, the embodied energy to complete or manufacture an object, and the wasted energy released are all important to conservation and obtaining an efficient and effective use (quality) of energy. If the regulation of energy processes is a concern in terrestrial habitation, it should be even more so for extra-terrestrial habitation where there is little margin for waste of any sort.
\end{abstract}

\section{Nomenclature}

$\Delta T=$ difference in temperature

PEL $=$ Peak of Eternal Light: hypothetical peak near a lunar pole with uninterrupted solar access

\section{Introduction}

SUSTAINING a habitat on the lunar surface, for any duration, is highly dependent upon making the most of the Slimited materials we can transport from Earth. Though there is abundant energy on the Moon, manufactured materials are necessary to capture, store, and convert it into useful forms.

Humans and their food require a balanced conditioned environment - thermal and luminous - during the lunar diurnal cycle. Except for small areas near the Lunar poles, the lunar night entails 354 hours with no solar exposure. The temperature extremes range from nearly $400^{\circ} \mathrm{K}$ during the lunar day to only $100^{\circ} \mathrm{K}$ at night (Williams, 2010). The lunar night is nearly 600 times longer than the eclipse period typical of Low Earth Orbit (Crane, 1991). Energy collection and long-term storage are therefore critical in the design of lunar habitats.

Moreover, designers should consider the energy invested in lifting components from Earth in relation to their eventual energy value on the Moon. To maximize the return on any energy investment, designers should consider the quality of available energy, its physical or molecular state or potential, and the type of energy required to perform a particular task.

This paper applies the concept of exergy to the design, procurement, and operation of lunar-based habitats. A total life-cycle evaluation of efficient and effective energy use must include the principles of exergy, the embodied energy of a material, and the theoretical potential of an energy source. It may be possible to develop a "usefulness vs. cost" factor for energy system components emplaced on the Moon, relating the potential work output to the

\footnotetext{
${ }^{1}$ Associate Professor, School of Architecture and Building, Waterfront Campus.

${ }^{2}$ Research Computer Specialist, UM3D Lab, Digital Media Commons. AIAA Senior Member.
} 
energy invested in the emplacement. The construction and operation of lunar habitats should consider and develop a total evaluation of energy processes if they are ever to claim "sustainability."

It has been suggested that energy supply and storage might be much less of a concern for a base at one of the socalled Peaks of Eternal Light (PELs) near one of the lunar poles. Though this is a slight digression from the main theme of this paper, it is worth considering.

Because the Moon's rotational axis is tilted only about $1.5^{\circ}$ from the ecliptic axis, versus $23.5^{\circ}$ for Earth's axis, a moderate peak near one of the poles might have nearly uninterrupted solar access throughout the months and years. The existence of such sites is mostly speculative, though candidates have been identified on the rim of the Shackleton Crater near the Moon's south pole (Kruijff, 2000). There are several things to consider before restricting lunar base studies to such sites:

- One should use the available energy wisely, even on a PEL.

- If PELs exist at all, they are few and small.

- As on Earth, a point within the Moon's polar circles (at a latitude beyond $\pm 88.5^{\circ}$ degrees), below the altitude necessary for a PEL, will experience winter darkness much longer than 354 hours.

- Because of the Moon's small axial tilt, the day-night diurnal cycle varies much less with latitude and season on the Moon than on the Earth. For the vast majority of the lunar surface, one must plan for a period of 354 hours, on average, every month, with no solar exposure; that is the rule, not the exception.

\section{Background to Energy, Exergy and Entropy}

Energy exists in many forms, each with its own unique characteristics and potential uses: electromagnetic, chemical, gravitational, nuclear, and thermal energy, to name a few. The concept of energy is familiar and obvious to us today, yet difficult to define precisely. Energy is a scalar quantity that cannot be observed directly but can be recorded by indirect measurements. Absolute energy is difficult to measure, but changes in energy are relatively easy to evaluate (Dincer, Rosen, 2007). Too often we use the term "energy" loosely, instead of defining it according to its potential and quality.

Thermodynamics plays a key role in the analysis of processes, systems, and devices in which energy transfers and transformations occur. The industrial revolution was driven by the discovery of how to exploit energy in converting heat into work. Nature allows the conversion of work into heat, but heat cannot be entirely converted into work (Dincer, Rosen, 2007).

The First Law of Thermodynamics is the law of conservation of energy, which states that energy is never made or lost but merely changes form. This law provides no information about the inability of any process to convert heat into mechanical work. A principle to explain the inefficiencies in energy processes, due to irreversibility, is the Second Law of Thermodynamics. This law deals with the exergy-entropy processes of an energy system.

The exergy of an energy form is a measure of its usefulness or quality or potential to cause change (Rosen, Dincer, 2001). Exergy explicitly accounts for "what is consumed." All systems, not only engineering systems but also biological systems including the human body, abide by the exergy principle: consuming food and thereby generating entropy, and disposing of the entropy into the environment (Shukuya, Hammache, 2002).

Entropy is a measure of energy quality. High entropy is an indication of great disorder. Therefore, high exergy implies low entropy. Entropy is a physical quantity that increases whenever energy or matter disperses while, at the same time, the total amounts of energy and matter are conserved (Shukuya, Komuro, 1996). When part of an energy process transfers into heat, this heat is a dispersion of energy and entropy is generated. In order to keep the entropy of a system constant, any additional generated entropy must be discarded into the environment.

Figure 1 shows an example based on Solberg (2008). Chemical energy enters the system at the top-left corner of the figure at a rate of 100 watts. A combustion turbine directly drives an electric generator. Only 32 watts go into the drive shaft, while 68 watts pass out of the turbine as exhaust. Of the 32 watts, 2 are lost as waste heat, leaving only 30 watts of electrical power. However, the 68 watts of exhaust are routed through a heat exchanger, which boils water to drive a steam turbine that ultimately contributes another 35 watts of electrical power, for a total of 65 watts electric emerging at the top-right. Exhaust heat from the steam turbine is put to further use lower in the figure. What is of interest here is that the state of energy quality can be considered (and calculated) when required to perform specific tasks.

Figure 1 is an indication of what exergy implies in terms of the thermal or energetic processes required of a task. Processes that use electricity for low exergy tasks such as low thermal heating are often wasteful and ill-considered. Electricity should be expended only where it is explicitly required or there is no alternative - such as electronics or electromechanical equipment. 


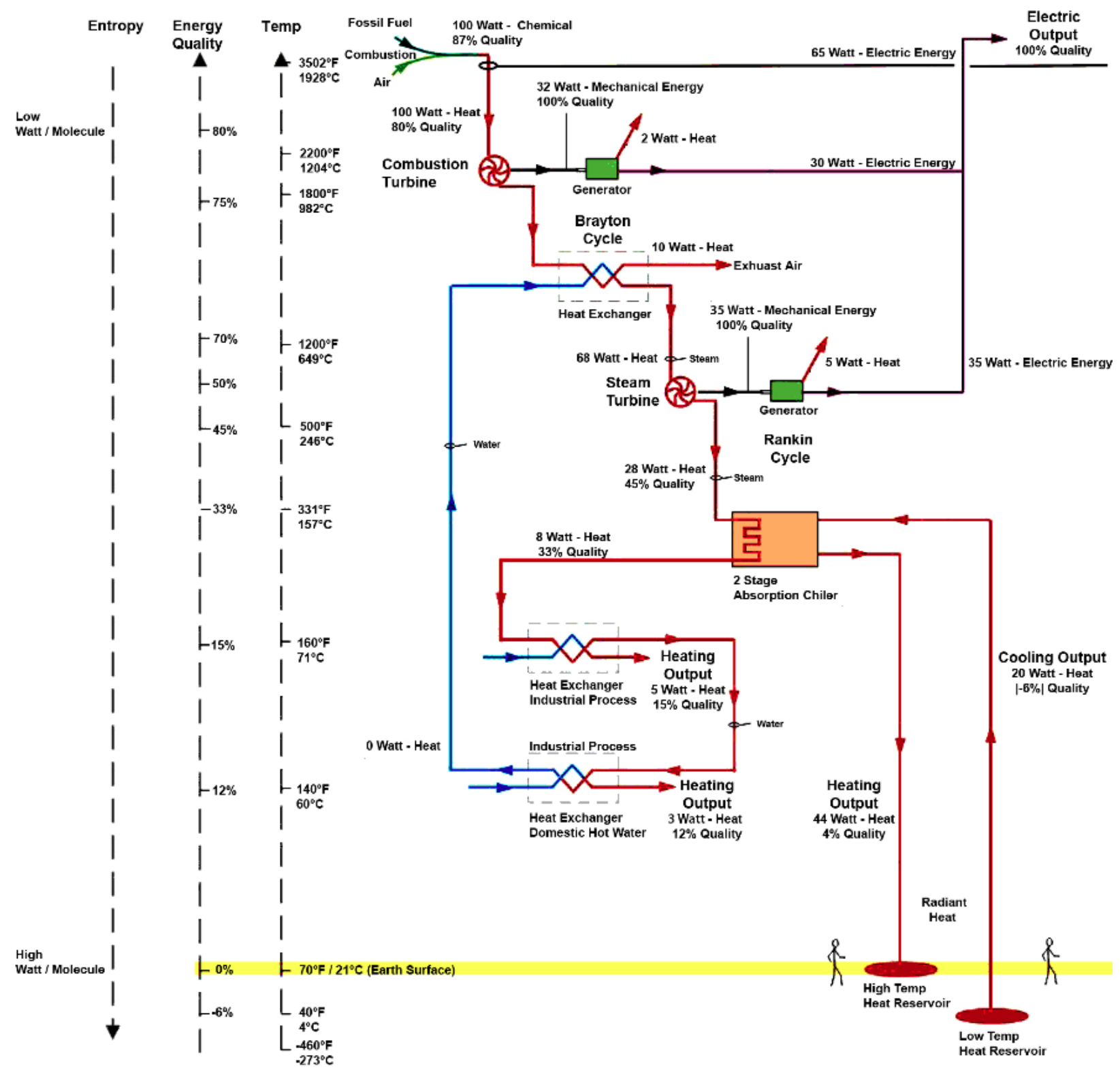

Figure 1. The state of energy quality required to perform various tasks (Solberg, 2008).

\section{Terrestrial Examples of Low Exergy and High Performance}

Habitats (buildings) often require low-grade energy (low exergy) for many of their operations. For example, high-pressure steam required for electrical power generation has a higher exergy than warm water needed by a dishwasher. The exergy-conscious approach entails matching the quality of energy supply and demand, in order to maximize the utilization of high value energy resources and minimize the irreversible dissipation of low-value energy into the environment (IEA, 2008).

\section{A. Passive Solar Heating}

When solar energy enters a room through a window and is absorbed partially into a concrete slab, stored and released into the room as heat, a classical example of the exergy-entropy process takes place. The Second Law of Thermodynamics states that there is a degradation of energy due to the irreversibility of all real processes. This law requires an exergy balance in the analysis and design of thermal systems. The entropy flowing from the concrete 
floor surface to the room air and back toward the glass window is larger than the incoming solar, due to the absorption at the floor surface. All the generated entropy, which originates from the consumption of solar exergy, is finally discarded into the outdoor environment through the uninsulated floor and the glass window (Shukuya, Komuro, 1996).

\section{B. Thermal Energy Storage Tank}

In a buried thermal storage tank, a hot medium flows through a heat exchanger within the tank and heat is transferred to the storage medium. Later in time a cold fluid is run through the tank and heat is transferred form the storage to the cold fluid. The amount of heat recovered to the cold fluid depends on the $\Delta T$ between the storage and the cold fluid as well as its time passing through the heat exchanger. Another consideration is the amount of heat that has escaped from the storage tank to the surrounding soil. A problem arises in evaluating the efficiency of the storage because lengthening the time that the recovery fluid is circulated can apparently increase the energy efficiency over an initial period. However, this neglects the fact that the storage temperature is continually decreasing as heat is dispersed to the surrounding soil. Eventually, little exergy is recovered, reflecting the fact that heat at near environmental temperatures does not make storage more thermodynamically efficient (Rosen, 2005).

\section{Power Plant}

Consider the electricity generated using an actual power plant (Rosen, 2005), illustrated in Figure 2.
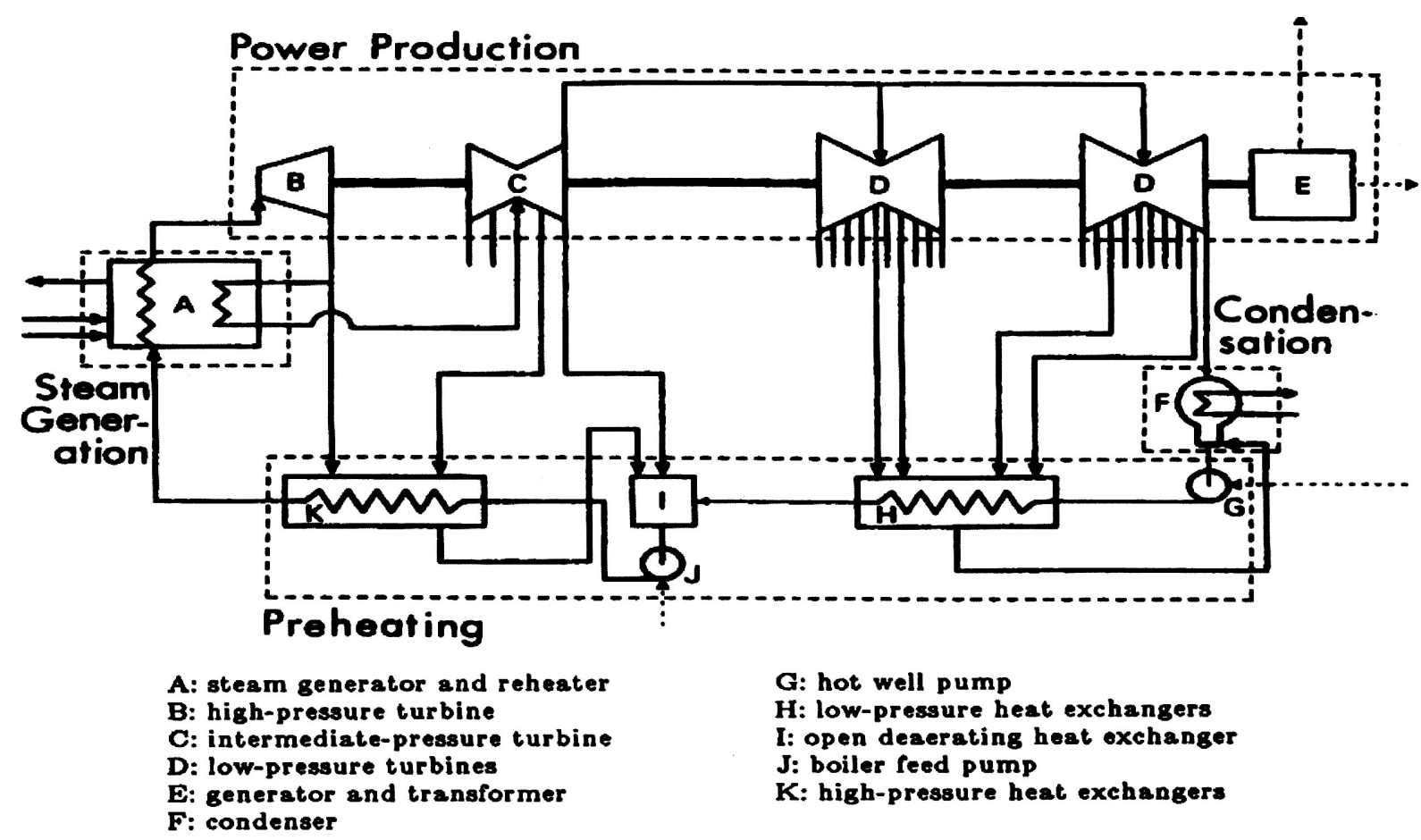

F: condenser

Figure 2. A breakdown of an electrical power plant (Rosen, 2005). The inputs are coal and air. The outputs for unit $A$ are stack gas and solid waste. The outputs for unit $E$ are electricity and waste heat. Electricity is input to units $G$ and $J$ and cooling water enters and exits unit $F$.

a. The plant uses steam generators which combust coal to produce primary and reheat steam.

b. The steam passes through a turbine driving an electric generator and transformer. Steam exhausted from the high-pressure turbine cylinders is reheated in the steam generator. Several steam extractors from the turbines preheat feed water in low and high-pressure heat exchangers.

c. Condensers receive the exhaust from low-pressure turbines, where cooling water condenses the steam.

d. Preheating heat exchangers and pumps increases the temperature and pressure of the condensed steam. 
The overall energy balances are depicted in Figure 3. The energy / exergy analysis is explained in greater detail by Rosen (2001). Several findings help to explain the thermodynamic behavior of the plant and identify areas having significant potential for efficiency-improvement:
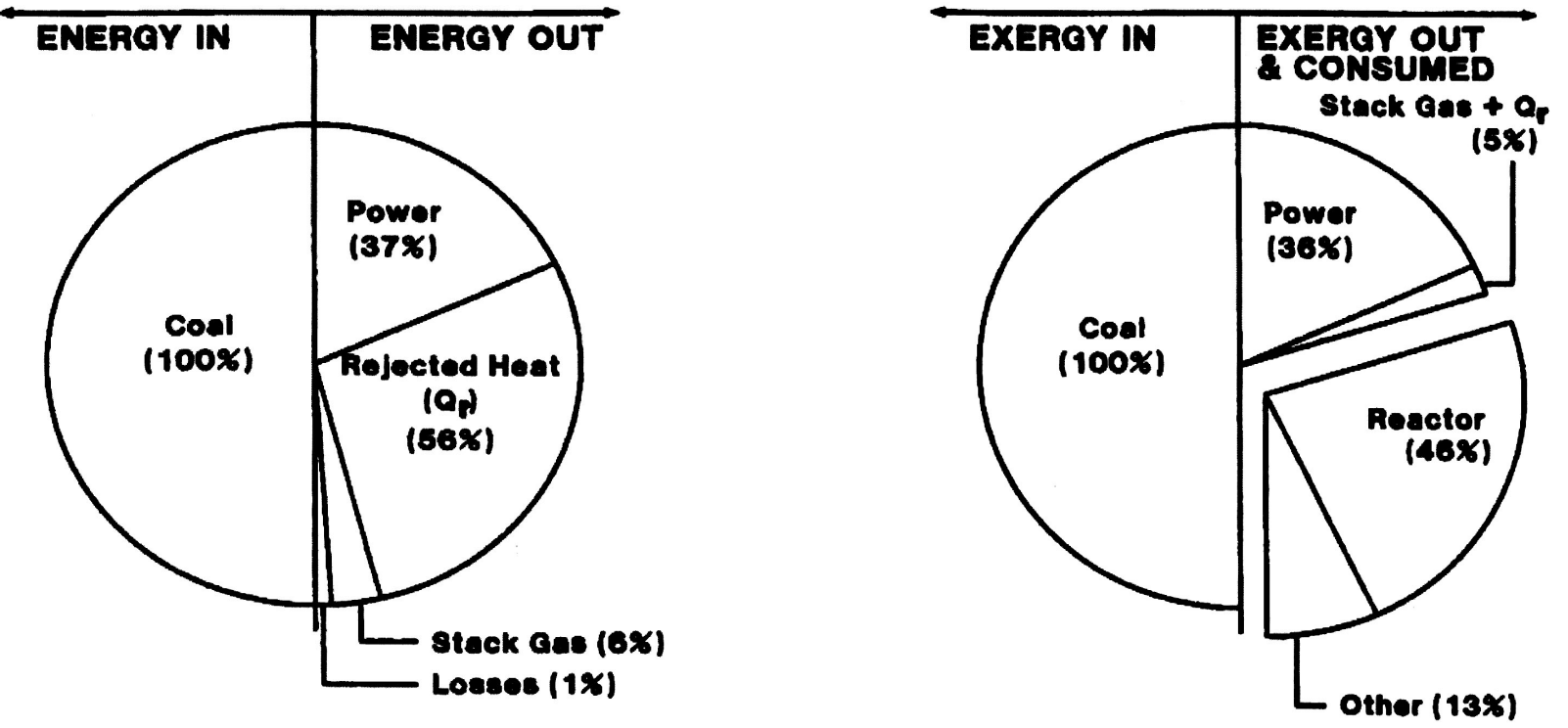

Figure 3. Overall energy and exergy balances for the power plant in Figure 2 (Rosen, 2005).

a. The overall energy efficiency (ratio of net electrical to coal energy) is $37 \%$ and the corresponding exergy efficiency is $36 \%$.

b. The steam generators appear significantly more efficient on an energy basis $(95 \%)$ than on an exergy basis (50\%). Physically, this discrepancy implies that, although most of the input energy is transferred to the preheated water, the energy is degraded as it is transferred. Most of the exergy losses in the steam generators are associated with internal consumptions (mainly due to combustion and heat transfer).

c. Large quantities of energy enter the condensers (about $775 \mathrm{MW}$ per unit) of which close to $100 \%$ is rejected. A small quantity of exergy enters (about $54 \mathrm{MW}$ per unit) of which about $25 \%$ is rejected and $75 \%$ internally consumed.

d. Energy losses in other plant devices were found to be very small (about $10 \mathrm{MW}$ total), and exergy losses (150 MW total) are almost completely associated with internal consumptions.

The above examples illustrate what can and should be considered in a complete thermodynamic analysis of an energy process. They demonstrate where inefficiencies in energy generation occur and can be improved.

\section{Considering Exergy for Lunar Habitats}

A lunar base will require substantial reliable power, continuous day and night. However, not all of the power demands are inherently electrical. In particular, heat and light might be obtained by other means - including as opportune by-products of electric power generation and recovery. According to the principles of exergy, it is important to consider the available type and ultimate use for power. No doubt, electrical power is needed to operate electromechanical, computing, and telecommunications equipment.

Landis (1989) summarized various concepts for capturing and storing solar power for the lunar night, including: photovoltaic and solar-dynamic collectors; electrochemical storage (batteries and fuel cells); capacitive and inductive storage; potential energy storage (e.g., raising and lowering masses of regolith via motors and generators); kinetic energy storage (e.g., flywheels); and thermal energy storage. His analysis of the thermal option, consistent with the others, focused on the potential for recovering electrical power, though it acknowledged that "only a fraction" of the stored energy would be accessible in that form. It also noted that electric power might be recovered 
from the waste heat of high-temperature manufacturing processes (such as magma electrolysis or glass manufacturing).

What is interesting for its absence is any discussion of the usefulness of the heat directly, versus converting it to electricity that might subsequently power electric heaters. Such an analysis would require a lunar base design that itemized not only the total quantity of power required, but its constituent types and qualities as well. Because the generation and use of electricity invariably release heat, first attention should be placed on the efficiency of essential electrical systems. Effort should then focus on the recovery and use of this heat for thermal storage, habitat conditioning, cooking, cleaning, sterilizing, and so on. Electric power for heating should be accounted last, and only to make up for a demonstrated deficiency of heat from other sources.

Crane and Dustin (1991) studied solar dynamic power to support lunar oxygen production. Much of the attraction for this power system was the substantial efficiency that might be realized by obtaining process heating directly, without intermediate conversion to and from electrical power. Crane (1991) followed up with an in-depth evaluation of in-situ thermal energy storage, independent of the ultimate application of the stored heat. He concluded that following a start-up period of about 4 months, periodic thermal losses from in-situ storage could be limited to about $4 \%$ of the energy input. He estimated the overall system efficiency (including the solar concentrators and heat exchangers) to be about $26 \%$ (e.g., $192 \mathrm{~kW}$ input for 14.75 days, versus $25 \mathrm{~kW}$ output for 29.5 days) - "comparable to alternative power technologies."

Motivated in part by Crane's work, Richter (1993) devised an experimental evaluation of "Lunar Thermal Energy from Regolith (LUTHER*')", using Minnesota lunar simulant. He acknowledged that the difference in surface gravity between the Earth-bound experimental apparatus versus the lunar surface might impact the convective heat transfer of molten regolith, but anticipated that sensible heat transfer would correlate closely.

These studies focused on the technical feasibility and predicted performance of lunar thermal storage, rather than the ultimate use of the stored energy. Though its potential application to high-temperature manufacturing processes was a motivating factor, lower-temperature applications for habitation and life support were not considered. Also omitted was any discussion of lunar regolith as a cold heat sink, for cooling a habitat. Though there may be abundant black sky for radiating unwanted heat, convection and conduction are more effective, and might offer more opportunity to recover the heat for later use.

These studies provide a useful technical foundation that designers ought to consider and extend by carefully documenting lunar habitat energy needs and devising efficient systems to meet them.

\section{Further Considerations}

An overall lifecycle accounting of energy inputs and outputs for a lunar base ought to include the energy invested in lifting components from the Earth to the Moon. This can be expected to strongly favor the use of in-situ energy and material resources, if they can be marshaled with minimal processing (and processing equipment lifted from Earth). Every additional kilogram of payload has an exponential impact on the energy invested in its launch and emplacement, and this should be factored into the exergy analysis.

Landis (1989) reviewed the specific energy capacities of various storage options. Table 1 summarizes his findings. He anticipated a very high energy density for lunar thermal storage. Since it utilizes mass already on the Moon, it may be expected to significantly reduce the energy expense of emplacing an energy storage system there. However, this table doesn't account for the overhead of launching the requisite construction equipment and other system components. A more detailed design and development plan must consider the energy invested in that, amortized over the expected useful life of the system. Other systems with less anticipated energy density may benefit somewhat from being fully constructed on Earth and not requiring the launch of any significant construction equipment.

Thus, as lunar bases develop past the initial period of bootstrapping, the profile of an optimum lunar habitat energy system will evolve to take advantage of emerging capabilities with on-site manufacturing from in-situ resources.

\footnotetext{
${ }^{*}$ No relation to the author.
} 
Table 1. Specific energy capacities of various storage options (Landis, 1989).

\begin{tabular}{|c|c|c|c|}
\hline \multirow[t]{2}{*}{ Storage Type } & & \multicolumn{2}{|c|}{ Specific Energy $(W \cdot h r / k g)$} \\
\hline & & Demonstrated & Anticipated \\
\hline \multirow[t]{2}{*}{ Electrochemical: } & Batteries: & 32 & 150 \\
\hline & Fuel Cells: & 50 & 1500 \\
\hline \multirow[t]{2}{*}{ Electromagnetic: } & Capacitors: & 10 & 20 \\
\hline & Inductors: & 0.5 & 100 \\
\hline Kinetic Energy: & Flywheels: & 20 & 35 \\
\hline Potential Energy: & Gravity: & - & $<1$ \\
\hline \multirow[t]{2}{*}{ Thermal: } & Phase-change material: & - & 250 \\
\hline & Lunar regolith: & - & 10000 \\
\hline
\end{tabular}

\section{Conclusion}

There is abundant energy on the Moon to power a habitat during daylight. The problem is to capture and convert that energy into useful forms, and to store and subsequently extract enough surplus energy to get through the night. Each of those stages requires the manufacture and emplacement of various system components. The large investment of energy necessary to transport them from the Earth to the Moon should motivate designers to strive for maximum efficiency.

It behooves architects to consider the overall efficiency of the system with respect to not only the quantity of energy needed, but also its quality. Not all energy needs are inherently electrical, and there are losses and inefficiencies in converting other forms of energy to and from electricity. A careful accounting of energy quality (light, heat, mechanical) as well as quantity, and deliberate efforts to capture and re-use "waste" energy, may increase the overall efficiency of the emplaced system. Furthermore, that higher efficiency may allow a lower mass launched from Earth, resulting in more energy savings and even higher overall lifecycle efficiency. The effect is exponential.

Exergy analysis can support the development and selection of new technologies and concepts with the potential for lowering exergy consumption for built environments. When the demand for heating and cooling has been minimized, the low-exergy approach aims at satisfying the remaining thermal energy demand using only low quality energy.

There are nearly as many lunar base designs as there are designers. A specification for yet another base is mission-dependent and beyond the scope of this paper. Rather, this paper aims to motivate designers who are "on a mission" to consider the potential benefits of exergy analysis to support the feasibility of their schemes.

\section{References}

Crane, Roger A. (1991). Evaluation of In-Situ Thermal Energy Storage for Lunar Based Solar Dynamic Systems (NASA CR189054). Cleveland, Ohio, USA: Lewis Research Center, National Aeronautics and Space Administration.

Crane, Roger A.; Dustin, Miles O. (1991). "Solar Dynamic Power / Process Heat Generation for the Proposed Lunar Oxygen Production Plant." 3rd ASME/JSME Thermal Engineering Joint Conference, Reno, Nevada, USA, 17-22 March 1991. [Cited by Crane, 1991.]

Dincer, Ibrahim; Rosen, Marc A. (2007). Exergy: Energy, Environment and Sustainable Development. Oxford, England, UK: Elsevier.

IEA (2008). "Low Exergy Systems for High-Performance Buildings and Communities.” (Energy Conservation in Buildings \& Community Systems, Annex 49.) International Energy Agency. http://www.annex49.com/background.html [cited 29 May 2010].

Kruijff, Michiel (2000). "The Peaks of Eternal Light on the Lunar South Pole: How They Were Found and What They Look Like." In B. H. Foing and M. Perry (eds.), Proceedings of the 4th International Conference on Exploration and Utilization of the Moon (ICEUM4) (ESA/ESTEC SP-462, p. 333-336). Noordwijk, The Netherlands: European Space Research and Technology Centre, European Space Agency.

Landis, Geoffrey A. (1989). Solar Power for the Lunar Night (NASA TM-102127). Cleveland, Ohio, USA: Lewis Research Center, National Aeronautics and Space Administration.

Richter, Scott W. (1993). Experimental Determination of In situ Utilization of Lunar Regolith for Thermal Energy Storage (NASA CR-191050). Cleveland, Ohio, USA: Lewis Research Center, National Aeronautics and Space Administration. 
Rosen, Marc A. (2001). "Energy- and Exergy-Based Comparison of Coal-Fired and Nuclear Steam Power Plants." Exergy, An International Journal (vol. 1, no. 3, p. 180-192). Paris, France: Elsevier Masson.

Rosen, Marc A. (2005). "The Role of Exergy in Increasing Utilization of Green Energy and Technologies." Proceedings of the International Green Energy Conference, Waterloo, Ontario, Canada, 12-16 June 2005.

Rosen, Marc A.; Dincer, Ibrahim (2001). "Exergy as the Confluence of Energy, Environment and Sustainable Development." Exergy, An International Journal (vol.1, no. 1, p. 3-13). Paris, France: Elsevier Masson.

Shukuya, Masanori; Hammache, Abdelaziz (2002 April). "Introduction to the Concept of Exergy - for a Better Understanding of Low-Temperature-Heating and High-Temperature-Cooling Systems." (Submitted to IEA Annex 37: "Low Exergy Systems for Heating and Cooling of Buildings.") VTT Tiedotteita - Research Notes 2158. http://www.lowex.net/downloads/ Introduction\%20to\%C9t\%20of\%20exergy.pdf [cited 29 May 2010].

Shukuya, Masanori; Komuro, Daisuke (1996). "Exergy-Entropy Process of Passive Solar Heating and Global Environmental Systems." Solar Energy (vol. 58, no. 1-3, p. 25-32). Amsterdam, The Netherlands: Elsevier.

Solberg, David (2008). "Energy and Exergy: the New Paradigm.” The Australian Building Regulation Bulletin. Canberra, ACT, Australia: Australian Building Codes Board.

Williams, David R. (2010 February). "Moon Fact Sheet.” Greenbelt, Maryland, USA: NASA Goddard Space Flight Center. http://nssdc.gsfc.nasa.gov/planetary/factsheet/moonfact.html [cited 29 May 2010]. 